CORRECTION

https://doi.org/10.1038/s41586-019-0905-0

\title{
Publisher Correction: Somatic APP gene recombination in Alzheimer's disease and normal neurons
}

Ming-Hsiang Lee, Benjamin Siddoway, Gwendolyn E. Kaeser, Igor Segota, Richard Rivera, William J. Romanow, Christine S.

Liu, Chris Park, Grace Kennedy, Tao Long \& Jerold Chun

Correction to: Nature https://doi.org/10.1038/s41586-018-0718-6, published online 21 November 2018.

In this Article, owing to an error during the production process, the top label in Fig. 5d read 'DISH 3/17' instead of 'DISH 3/16'. This error has been corrected online. 